Cinémas

Revue d'études cinématographiques

Journal of Film Studies

\title{
Fonctions et origines du bonimenteur du cinéma des premiers temps
}

\section{André Gaudreault et Germain Lacasse}

Volume 4, numéro 1, automne 1993

URI : https://id.erudit.org/iderudit/1000116ar

DOI : https://doi.org/10.7202/1000116ar

Aller au sommaire du numéro

Éditeur(s)

Cinémas

ISSN

1181-6945 (imprimé)

1705-6500 (numérique)

Découvrir la revue

Citer cet article

Gaudreault, A. \& Lacasse, G. (1993). Fonctions et origines du bonimenteur du cinéma des premiers temps. Cinémas, 4(1), 132-147.

https://doi.org/10.7202/1000116ar
Résumé de l'article

Le bonimenteur, cet " explicateur de vues » en direct, est devenu une figure quasi mythique de l'histoire du cinéma des premiers temps. Il avait, semblait-il, laissé peu de traces. Tant et si bien que, sur le plan international, on ne l'a redécouvert qu'il y a une quinzaine d'années. Mais des recherches plus poussées ont permis de découvrir que, sur le plan national, il avait eu une importance plus grande qu'ailleurs. Il s'agira ici d'effectuer un survol historique des origines du bonimenteur, tout en tentant d'expliquer le rôle et les fonctions de cet adjuvant narratif dont on commence enfin à comprendre qu'il fut essentiel, du moins au Québec, pour l'exploitation locale de films qui, presque sans exception, venaient d'ailleurs. 


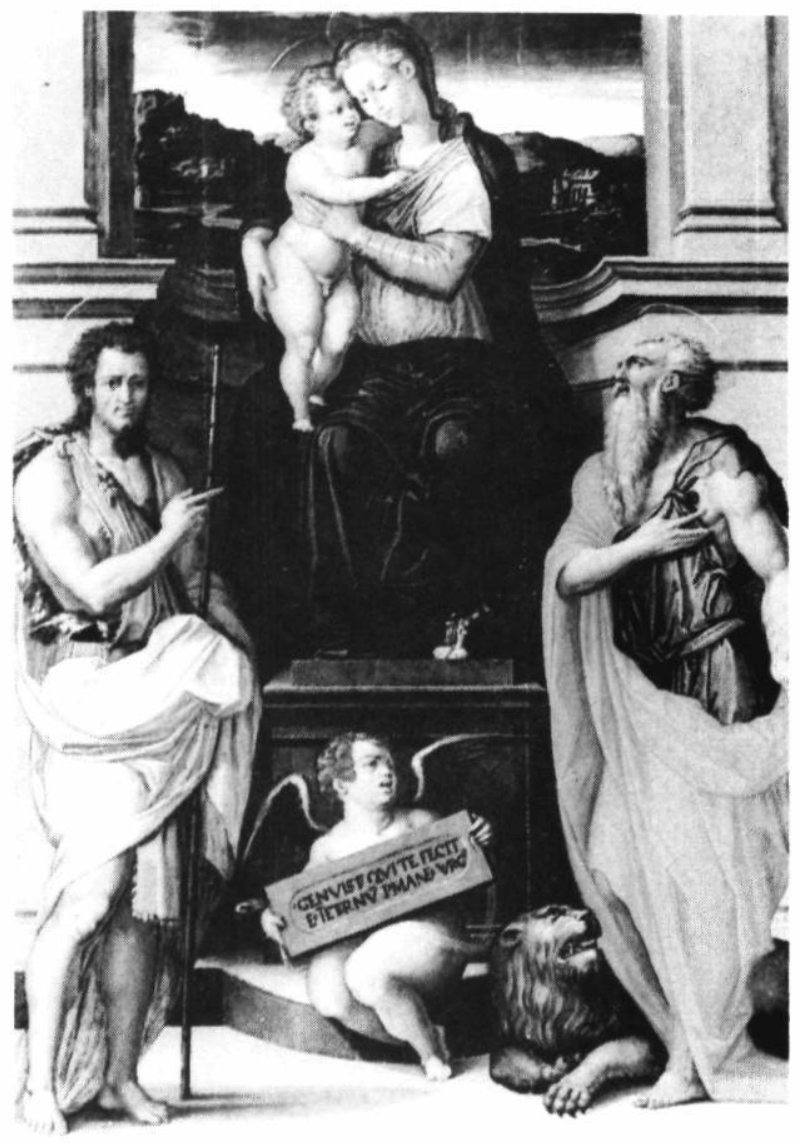

Madone à l'Enfant avec Saint Jean-Baptiste et Saint Jérôme, huile sur panneau de Giorgio Vasari (1511-1574)

De l'admoniteur... au bonimenteur... 


\title{
Fonctions et origines du bonimenteur du cinéma des premiers temps
}

\section{André Gaudreault}

Collaboration : Germain Lacasse

\section{RÉSUMÉ}

Le bonimenteur, cet «explicateur de vues» en direct, est devenu une figure quasi mythique de l'histoire du cinéma des premiers temps. Il avait, semblait-il, laissé peu de traces. Tant et si bien que, sur le plan international, on ne l'a redécouvert qu'il y a une quinzaine d'années. Mais des recherches plus poussées ont permis de découvrir que, sur le plan national, il avait eu une importance plus grande qu'ailleurs. Il s'agira ici d'effectuer un survol historique des origines du bonimenteur, tout en tentant d'expliquer le rôle et les fonctions de cet adjuvant narratif dont on commence enfin à comprendre qu'il fut essentiel, du moins au Québec, pour l'exploitation locale de films qui, presque sans exception, venaient d'ailleurs.

\begin{abstract}
The barker, a man who "explains the moving pictures," bas become a quasi-mythical figure from the earliest period of cinema history, one that seems to have left few traces. Because of this it was only fifteen years ago that he was rediscovered internationally. But further research has revealed that he had an even greater national importance in Quebec than elsewhere. This is a study of the origins of the barker and, at the same time, an attempt to explain the role and functions of this narrative adjunct whose existence, we are beginning to see, was essential, at least in Quebec, for the marketing of films that, almost without exception, came from elsewhere.
\end{abstract}


Dans un ouvrage publié il y a maintenant cinq ans ${ }^{1}, \mathrm{j}^{\prime}$ essayais de démontrer que le dispositif narratif du cinéma mettait à contribution, à la fois, ces deux modes fondamentaux de communication narrative que sont la «diégèsis mimétique» (la diègèsis dia mimèseôs ou récit par voie d'imitation de Platon ${ }^{2}$ ) et la «diègèsis non mimétique» (la haplè diègèsis ou récit simple). L'hypothèse que j'y développais était à l'effet que la communication d'un récit par le film impliquait la superposition de deux couches distinctes de narrativité, la première relevant de ce que j'ai alors suggéré d'appeler la monstration, définie comme corollaire des opérations de "mise en scène» et de «mise en cadre» (soit, en gros, le tournage), et la deuxième de la narration, corollaire celle-là des opérations de "mise en chaîne» (soit, en gros, le montage). Je faisais aussi écho, dans le livre, à l'activité, essentielle pour le cinéma des premiers temps, de cette figure quasi mythique du bonimenteur, véritable «explicateur de vues en direct», dont on venait à peine de découvrir l'existence, au tournant des années $80^{3}$.

Ce bonimenteur, qui avait pour mission d'accompagner de son discours verbal la projection des "vues animées», apparaissait d'ailleurs aux chercheurs comme une espèce de fantôme aux contours relativement flous. En effet, les traces qu'il avait laissées dans les documents que nous avait légués l'Histoire semblaient vraiment trop parcellaires pour permettre à quiconque d'en tracer un portrait le moindrement fidèle. Ces traces se résumaient à quelques témoignages d'époque ainsi qu'à quelques textes fournis à l'exploitant par les sociétés de production, en même temps que les catalogues de films, pour constituer la base du boniment à livrer au public durant la projection ${ }^{4}$. Récemment, la situation s'est carrément modifiée avec, notamment, la découverte de nouvelles traces, nombreuses et détaillées, de l'activité fascinante exercée par cet «agent» de la narration filmique des débuts du cinéma.

Bien que j'aie consacré un chapitre entier de mon livre au bonimenteur (le Chapitre XII intitulé «Un spectacle monstratif à narration assistée») et au système narratif que son interaction avec le film supposait, j'étais alors loin de me douter que le bonimenteur avait pu jouer un rôle d'une importance aussi grande que celui qu'il a effectivement joué, au Québec du moins ${ }^{5}$. Alors que, dans les autres pays (sauf le Japon, bien entendu 6 ), le bonimenteur semble être disparu, petit à petit, autour de 1908, on peut facilement suivre sa trace au Canada, au Québec surtout, jusque dans les années 20. Il s'agit là d'une donnée essentielle relative à l'exploitation du cinéma en sol 
canadien, et qui lui donne une spécificité hors du commun, surtout si l'on tient compte, en sus, de la présence massive du bonimenteur, plus massive qu'ailleurs semble-t-il, dès les toutes premières années du cinéma (les premières traces qu'il nous a laissées, au Québec, remontent à 18977).

Nous avons en effet pu accumuler une série assez impressionnante de données relatives à l'activité du bonimenteur au Canada français, principalement par le dépouillement systématique des journaux. Nous savons notamment que le premier bonimenteur qu'ont entendu les Québécois est un dénommé Henry de Grandsaignes d'Hauterives ${ }^{8}$, un aristocrate breton ruiné qui, dès 1897 , tenta de faire fortune avec le cinéma naissant. Nous savons aussi que, la première année, celui-ci donnait ses explications non pas durant la projection elle-même, mais plutôt, dans le cas des films «uniponctuels 9 », entre deux films, ou encore, dans le cas des films "pluriponctuels 10 », entre deux scènes (ou deux plans, ce qui revenait à peu près au même à cette époque). Nous savons aussi que, dès 1898, il sera amené à changer sa technique : «M. le vicomte d'Hauterives donne maintenant les explications au fur et à mesure que les tableaux vivent devant les yeux», comme nous l'apprend le chroniqueur d'un hebdomadaire régional («L'Historiographe», Le Courrier de St-Hyacinthe, 29 octobre 1898). Le travail d'Henry de Grandsaignes fit à plusieurs reprises l'objet de commentaires de la part des journalistes québécois. Ainsi, à titre d'exemple : «Nous devons dire que M. le vicomte d'Hauterives nous fait le récit de chaque vue avec clarté, précision et délicatesse. Aussi l'auditoire l'écoute avec attention. Il sait le captiver et l'intéresser» («Dans les Bois-Francs», L'Écho des Bois-Francs, Victoriaville, 3 mars 1900); le film est accompagné par un «homme qui se morfond à vous expliquer ce que vous voyez, que vous n'écoutez pas et qui vous récite imperturbablement son boniment jusqu'à la fin» (Raoul Labrosse, «Le Cinématographe», Les Annales térésiennes, Ste-Thérèse, novembre 1900)!; "the entertaining descriptions [were] in exquisite French" («Historical Cablo», Quebec Daily Mercury, Québec, 30 avril 1898); «chaque syllabe souligne un [j]eu de physionomie ou de scène» («L'Historiographe à Joliette», L'Étoile du Nord, Joliette, 31 janvier 1901) ${ }^{11}$; etc.

Les commentaires journalistiques recueillis sur l'activité du bonimenteur au Canada français sont très précieux puisqu'ils représentent les seuls témoignages sur les modalités d'opération d'un agent essentiel de la narration filmique à l'époque du cinéma des premiers temps. D'autant que la prestation du 
bonimenteur, on l'aura compris, est aussi fugace qu'une représentation théâtrale, sinon plus encore, du fait qu'elle est toujours, peu ou prou, le fruit de l'improvisation, au contraire de la pièce de théâtre dont le texte est, plus souvent qu'autrement, consigné par écrit. En effet, les indices que nous avons recueillis nous permettent d'avancer que les boniments semblent avoir été le plus souvent le fruit d'une improvisation relativement libre. L'envoi, par les sociétés de production, de feuillets d'«instructions» à l'intention du bonimenteur (ou du "conférencier», ou «bonnisseur» - lecturer en anglais comme on disait plutôt à l'époque), sous forme de "récits écrits», est apparemment resté une pratique relativement peu commune $^{12}$. Le spectacle filmique bonimenté est intéressant pour nous dans la mesure où il représente un cas assez original de juxtaposition syncrétique, proprement «intermédiatique», d'une prestation relevant de la "diégèsis mimétique» (ces récits par voie d'imitation que sont les films) à une prestation procédant de la «diègèsis non mimétique» (ces récits simples que distille verbalement le bonimenteur), soit une combinaison et un brassage, assez singulier (mais loin d'être unique comme nous le verrons dans la suite de cet article), de monstration iconique et de narration verbale.

Un peu après le tournant du siècle, cet «explicateur de vues» qu'est le bonimenteur était devenu une nécessité dans le cas notamment de certains films pluriponctuels plus ambitieux, ne serait-ce que pour injecter de la narration dans ces images mouvantes muettes et résolument monstratives qui constituent l'ordinaire du cinéma des premiers temps. L'arrivée de la "pluriponctualité», qui était une nouvelle structure permettant entre autre de donner de l'expansion aux récits, avait en effet causé autant de problèmes narratifs qu'elle pouvait en avoir résolu. Au premier chef, le problème de ce qu'on appelle communément la «continuité narrative». Celle-ci, qui était assez aisément obtenue par la monstration en un seul plan (il n'y a rien, au fond, d'apparence plus continue qu'une suite de photogrammes projetés), se voyait menacée par la variation angulaire et la modulation spatio-temporelle que suppose la pluriponctualité : chaque hiatus, chaque saute de caméra pouvait donner lieu à des hiatus spatiaux et temporels, creuser des trous dans le tissu narratif, rompre le fil du récit et laisser une part trop grande chez le spectateur à l'interprétation, quand ce n'était pas à l'incompréhension. Le bonimenteur avait ainsi diverses fonctions dont celles d'expliquer au spectateur le sens des images montrées, de lui indiquer les relations et les liens entre ces 
personnages que l'on voyait certes sur l'écran mais dont on n'arrivait pas à entendre la voix. C'était une sorte de "Grand Parleur» (équivalent verbal du fameux "Grand Imagier» cinématographique) qui, installé dans la salle avec les spectateurs, commentait les images animées et, surtout, narrait les événements de cette histoire à raconter, en ajoutant sa narration «scénique» à la monstration iconique du film muet.

Le bonimenteur a des origines qui datent d'avant le cinéma. En effet, les images muettes des représentations de lanterne magique, privées comme publiques, étaient, règle générale, accompagnées d'un commentaire narratif et explicatif de la part d'un «conférencier». La plus importante manifestation «grand public» de la lanterne magique fut vraisemblablement son utilisation par des prédicateurs qui montaient de véritables spectacles audiovisuels de propagande religieuse. Le sujet le plus récurrent de pareilles présentations, c'étaient, bien entendu, la Vie et la Passion du Christ ${ }^{13}$. De tels spectacles étaient composés d'une trame visuelle assurée par une quantité parfois impressionnante d'images fixes (des plaques le plus souvent photographiques ${ }^{14}$ ), projetées par une lanterne magique, représentant une Vie et Passion du Christ accompagnées notamment (notamment, parce qu'il pouvait aussi y avoir de la musique) d'un commentaire en direct de la part d'un présentateur dont le discours verbal se faisait fort de concurrencer la trame narrative des seules images. La Passion fut d'ailleurs l'un des thèmes les plus récurrents des premiers films et des premières représentations cinématographiques. Les premiers spectacles bonimentés sur la Passion du Christ, qui ont été légion les premières années, devaient beaucoup à la religion et à ses traditions de représentation. Sans être aussi importante qu'elle le fut pour le théâtre qui, on le sait, est né des cérémonies religieuses qui avaient cours dans la Grèce antique et dans l'Europe catholique médiévale, l'influence de la religion dans le développement du cinéma reste, pour le moins, considérable. Ainsi du bonimenteur-conférencier, qui ressemble au prêcheur sur plus d'un plan. Lorsqu'il commente la Passion, le bonimenteur est en effet dans une position assimilable à celle du prêcheur qui commente les Évangiles. Il actualise un récit déjà connu, transmis par la tradition orale et écrite, comme il le fait d'ailleurs pour plusieurs autres films des premiers temps ayant des thèmes plus profanes (Ali-Baba, Cendrillon, La Case de l'oncle Tom, etc.). Il s'agissait de récits que le spectateur connaissait déjà et dont il pouvait, à la limite, tout de même 
comprendre certains éléments sans l'aide d'un adjuvant narratif comme le bonimenteur.

L'Histoire a cependant connu, bien avant l'époque de la lanterne magique, d'autres types de "présentateurs» ou "commentateurs» verbaux ayant la tâche d'officier à titre de narrateur d'une prestation actorielle représentant la Passion du Christ (ou autre «mystère» de la foi). Ainsi du festaiuolo du Moyen Âge, ce maître de cérémonies qui accompagnait de son discours verbal les fameux Mystères médiévaux et dont le rôle était de mettre du liant, du liant narratif vraisemblablement, entre les divers tableaux «ajourés» des Mystères. Comme le fait, lui aussi, le bonimenteur du cinéma des premiers temps, qui doit, pour favoriser une lecture continue des tableaux 15 du film qu'il montre, injecter, entre les images, le liant de son discours verbal. Les pratiques du genre se retrouvaient même, souvent, directement associées au culte religieux. Comme le rapporte Alain Laframboise :

Dès le XIVe siècle, le sermon et la liturgie étaient illustrés par des représentations dramatiques, dirigées par le prédicateur agissant à titre de festaiuolo. Le prédicateur annonçait, décrivait et interprétait, moralisait le jeu théâtral qui accompagnait son sermon lors des fêtes du Vendredi saint (p. 98).

Le festaiuolo médiéval avait lui aussi de qui tenir. La juxtaposition d'un commentaire verbal narratoriel à une prestation actorielle sur le thème de la Passion du Christ est en effet une pratique fort ancienne. Comme le rapporte Gaëtane Marsot :

Le festaiuolo lui-même descend d'une longue tradition scénique. Hermann fait remonter le personnage de l'«expositeurprésentateur» aux premiers siècles après la chute de Rome, alors que la comédie était lue par un poète à une chaire et jouée en pantomime par des acteurs derrière lui (p. 18).

Le festaiuolo et ses semblables n'ont bien sûr laissé que fort peu de traces de leur activité mais ils auraient eu, dans la peinture, une «descendance» qui nous est, elle, encore aujourd'hui directement accessible. Il s'agit de la figure relativement peu connue de l'«admoniteur»16 (appelé parfois, de façon plus traditionnelle, le commentateur ou, comme le propose un auteur récent, le «montreur»17), que l'on considère comme directement issu du festaiuolo ${ }^{18}$. C'est en effet au XVe siècle, alors que le festaiuolo était semble-t-il encore en exercice, que l'on trouve les premières traces de l'admoniteur, à la fois sur le 
plan théorique (dans le fameux traité d'Alberti, Della pittura ${ }^{19}$ ) et sur le plan pratique (dans certaines peintures de l'époque).

Cette figure proprement fabuleuse de l'admoniteur n'est pas très connue en dehors du cercle restreint des spécialistes. Il s'agit d'une figure peinte, un personnage ni plus ni moins, faisant partie du monde signifié par la toile, mais qui adopte une attitude et une posture qui font d'elle un genre d'intermédiaire entre le spectateur et le monde peint (voir les figures 1, 2 et 3 ). Comme on peut le constater, l'admoniteur fixe la plupart du temps son regard en direction du spectateur et a, souvent, le doigt tendu pour indiquer à ce dernier ce que doit être le point d'ancrage de son regard. L'un des aspects les plus intéressants de l'admoniteur réside dans cette faculté qu'il a, comme figure, de briser, par sa seule présence, l'unité du discours pictural. Son regard relativement spéculaire du regard du spectateur fait qu'il y a, dans l'énoncé pictural, coprésence apparente de deux discours. Celui de la toile dans son ensemble, un monde d'instances qui se donnent à voir, et celui de l'admoniteur, une instance qui, au contraire, donne à regarder.

À première vue, le bonimenteur du cinéma des premiers temps partage un certain nombre de fonctions avec l'admoniteur. Il apparaît évident, en tout cas, qu'admoniteur et bonimenteur servent tous d'eux d'«agent de liaison» et, même, de relais, entre l'«intra-pictural» et l'«extra-pictural». À la différence que le premier, l'admoniteur, fait ce relais à partir même de l'«intrapictural», tandis que c'est à partir de l'«extra-pictural» que le bonimenteur exerce ses fonctions. D'un côté, donc, l'admoniteur, qui est présent dans le texte et qui, acteur parmi les acteurs, est irréductiblement exclus de l'espace spectatoriel. De l'autre, le bonimenteur, qui fait le relais entre actor et spectator à partir même de l'espace spectatoriel dont il est, frontalement et irréductiblement, du moins sur le strict plan phénoménologique, partie prenante. Par ailleurs, ces deux figures de relais que sont l'admoniteur et le bonimenteur remplissent de semblables fonctions monstratives et commentatives. Ils sont tous deux placés de façon à pouvoir nous montrer du doigt ce qu'il faut regarder dans l'image ${ }^{20}$. Ce faisant, de par l'élection qu'ils font des éléments dignes de retenir notre attention, ils ont tous deux une fonction relativement commentative, et même, «évaluative». Bien sûr, la chose est plus évidente chez le bonimenteur, qui a, lui, recours à la parole, ce qui lui permet de remplir, en plus, une fonction narrative presque statutairement refusée à l'admoniteur. 
On peut aussi voir l'admoniteur et le bonimenteur comme des délégués, auprès du spectateur, de cette instance fondamentale responsable de la monstration que j'ai suggéré d'appeler le «monstrateur». En ce sens, admoniteur et bonimenteur ont une fonction de médiation entre monde actoriel et monde spectatoriel et l'on peut penser qu'ils agissent à titre d'adjuvants du monstrateur pour aider la toile ou l'écran à accoucher d'un sens. L'admoniteur et le bonimenteur sont donc en quelque sorte des délégués de cette instance supérieure qu'est le Grand Imagier, et on peut penser que, dès lors que leur discours est le moindrement tonitruant, ils évoquent assez directement la présence en sous-main de cette instance d'énonciation responsable de l'ensemble signifiant dont ils ne sont qu'une partie. Et le moins que l'on puisse dire, c'est qu'effectivement ces deux figures ont l'heur de tenir des discours qui tonitruent... Les traces ou marques d'énonciation pullulent en effet dans le discours de l'admoniteur et du bonimenteur. Pensons notamment au fait que, toujours ou presque, ces deux instances «interpellent» le spectateur et s'adressent «directement» à lui.

Il est intéressant de faire remarquer comment les caractéristiques de ces deux figures, notamment leur fonction de délégués d'une autre instance, semblent traverser les modes d'expression, les frontières, les époques et les genres. En Haïti par exemple, on retrouve une semblable hiérarchisation des instances narratives à la fois dans les contes, dans la littérature en général et même dans la peinture :

La structure narrative des récits romanesques reproduit la hiérarchisation des voix qui, dans le conte traditionnel, fait de la parole du narrateur présent l'écho de la voix d'un supernarrateur. Celui qui nous parle reproduit la voix d'un autre conteur. Il prend la place d'un autre pour tenir le discours de celui-ci. Ainsi l'histoire contée dans le présent redouble celle que le narrateur absent aurait contée. Cette structure de la narration, et forcément de la représentation, est si commune et si profondément enracinée chez les artistes haïtiens, qu'on peut la retrouver même dans la peinture populaire. Ce qui permet de parler de l'image comme écho, l'image picturale en effet se faisant le double, l'écho d'une voix, celle de la langue (Laroche, p. 67).

De par la nature même du média qu'il investit, la peinture, l'admoniteur présente cependant, à titre de représentant d'une instance absente, certaines caractéristiques qui lui sont propres. Il est toujours dans une position relativement «forcée», le doigt et le bras tendus. Il est souvent la seule figure de tout le tableau dont le geste semble avoir été comme saisi au vol. Son attitude tranche nettement avec le contexte qui l'entoure. On dirait qu'il 
«veut» nous parler, on dirait qu'il «va» nous parler. Mais, en bon personnage muet, l'admoniteur, ne pouvant joindre la parole au geste, se voit réduit à plutôt subsumer celle-là, la parole, par celui-ci, le geste, ayant ainsi recours à la plus étrange pantomime qui soit. Imaginez, une pantomime «figée», sans mouvement d'aucune sorte...

L'admoniteur fait, au fond, rupture dans une toile aux proportions perspectivistes par rapport auxquelles il ne cadre pas toujours, l'inscription du spectateur étant déjà prévue de façon maximale par l'équivalence structurale du «point de vue» et du «point de fuite». On pourrait ainsi penser que l'admoniteur vient plutôt casser l'aspiration éventuelle du spectateur dans le tableau, à l'instar du bonimenteur dont il faut attendre la disparition pour que le spectateur de cinéma se fasse lui aussi, à son tour, aspirer dans la diégèse du film qu'on lui projette. La position adoptée par l'admoniteur empêche en effet le spectateur de se laisser emporter dans la force d'attraction "vectorisante» du continuum latéral (gauche-droite) où loge la narrativité picturale. Placé à gauche, cas le plus fréquent, l'admoniteur s'impose au regard du spectateur en début de lecture du tableau, rencontre qui empêche de se noyer dans la narrativité du latéral un spectateur qui, suivant en cela l'invitation d'un admoniteur lui indiquant le chemin de la perspective, entre dans la profondeur pour y découvrir le sens du tableau. Un peu comme si, dans pareil cas, la monstration jouait contre la narration, le ponctuel contre le vectoriel. En fait, l'admoniteur semble, par nécessité, situé aux confins de la monstration et de la narration, au point de jonction entre le ponctuel et le vectoriel. Ainsi, non seulement sa position près du bord gauche de la toile l'amène-t-elle à s'offrir en pâture au lecteur dès le moment où ce dernier y pose son regard, mais encore est-il situé, plus souvent qu'autrement, au premier plan de l'action dépeinte, en relation proximale, donc, avec le spectateur, s'imposant encore une fois d'emblée à sa lecture. Même si la fonction première de l'admoniteur se situe clairement du côté de la monstration (n'est-il pas est un «anthropoïde» qui «montre» du doigt et invite à une lecture ponctuelle et axiale?), il n'en reste pas moins une véritable figure ambivalente puisqu'on peut voir en lui, tout aussi bien, un adjuvant de la narration. Ne serait-ce qu'en fonction de la verticalité inhérente à sa nature anthropomorphe qui, de par sa configuration, invite à une lecture latérale, vectorielle donc. Ne serait-ce que parce qu'il est toujours, ou presque, une instance intradiégétique, une instance actorielle. Certains admoniteurs, en effet, jouent même un rôle 
diégétique spécifique aisément identifiable, le plus souvent de seconde importance, ange-serviteur, dame de compagnie, etc.

Dans la mesure où son geste est, comme celui de l'admoniteur, d'abord et avant tout un geste de montreur, de monstrateur, le bonimenteur semble lui aussi situé aux confins de la monstration et de la narration. Ce qu'il montre cependant, ce sont des images animées toujours déjà vectorisées, au sein desquelles le ponctuel est certes relativement important, mais jamais totalement dominant. Par ailleurs, son don de parole, qui est refusé à l'admoniteur, lui permet de tisser des fils, les fils de la narration, entre les différents tableaux de la monstration. Ce don de parole lui permet, en plus, d'«interpréter» le film² ${ }^{21}$, dans les deux sens du mot (comme on dit «interpréter un rêve» et «interpréter une partition musicale»). La fonction du bonimenteur est tout à fait similaire à celle de ces "conteurs oraux» que toutes les sociétés ont connus à un moment ou à un autre de leur histoire et dont l'origine se perd dans la nuit des temps. Comme le conteur oral traditionnel, en effet, le bonimenteur «ne se contente pas de broder sur un thème donné [et] tout son talent consiste précisément à savoir adapter son récit à son auditoire» (VelayVallantin, p. 28).

Le bonimenteur fonctionne ainsi un peu comme le fait un guide dans un musée. Son rôle, à lui aussi, c'est d'amener les gens qui lui sont confiés à circuler dans un dédale de «tableaux» 22 , dans bien des cas selon un tracé qu'il impose «luimême» (le bonimenteur, c'est en effet souvent l'exploitant «luimême» et c'est l'exploitant qui, à cette époque, monte «luimême», selon l'ordre qui lui sied, les courts films qui composent son spectacle), et à partir d'une interprétation qui est et reste sienne, quand bien même il suivrait un tant soit peu l'orthodoxie en la matière (les textes fournis pour le boniment). Il est là, situé au seuil de la diégèse, pour montrer au spectateur la porte d'entrée donnant accès à ce monde imaginaire que les images animées font naître devant ses yeux. Il est là, comme un véritable appât humain, pour captiver le spectateur, au sens littéral du mot (Le Robert : "retenir captif; faire prisonnier»), pour l'attacher au récit. Francesco Casetti n'a-t-il pas écrit que le dispositif cinématographique est organisé comme un piège (p. 22)?

Comme le conteur, le bonimenteur retient l'écoute parce qu'il ressemble au spectateur, parce qu'il le connaît et parce qu'il sait comment actualiser pour lui le récit qui lui est offert. Bien sûr, l'«autorité» (noter qu'à l'origine de ce mot, il y a auctor, auteur) du bonimenteur, installé au seuil de la diégèse, confère au récit une crédibilité qu'il n'aurait pas eue autrement. Mais, justement, 
le bonimenteur est, et «reste», au seuil de la diégèse, comme le spectateur. Et, au fond, il s'interdit, et interdit au spectateur, d'y entrer véritablement. La distance créée par le commentaire du bonimenteur entre le monde diégétique mis sur film et le spectateur reste vraisemblablement irréductible. Il est le support d'un mode de narration qui se cherche encore, et qui n'a encore pas mis l'entrée «en fiction» du spectateur au plan de ses priorités. Les «diégèses» cinématographiques sont alors en effet des diégèses «filmées» plutôt que des diégèses «filmiques». Ainsi, par exemple, des Passions. Au contraire de ce qui allait bientôt prédominer à Hollywood, aucune d'entre elles ne présente ses images comme si elles originaient d'un monde clos et autarcique dans lequel pourrrait s'engouffrer le spectateur. Comme le remarque Noël Burch :

Aujourd'hui (et depuis plus de soixante ans déjà) l'adaptation à l'écran, dans le cadre du système dominant, d'une œuvre même très connue du grand public, doit «faire comme si» cette œuvre n'avait aucune existence antérieure, extérieure au film. Le «digest» qui en est fait doit «tenir debout» tout seul : une adaptation de type hollywoodien, même de la Bible, identifiera et campera tous les personnages et toutes les situations comme pour la première fois, selon les canons du monde clos, autarcique du roman bourgeois, où l'histoire (avec et sans majuscule) n'existe qu'en tant que le texte l'invente (p. 37).

Au contraire donc de l'adaptation hollywoodienne à venir, la Passion filmée atteste, dans la façon même dont elle est filmée, de l'existence du texte ou, plutôt, des textes qui l'ont précédée (les textes scripturaux du Nouveau Testament, les «textes» scénographiques d'Oberammergau, etc.). Elle reste une illustration filmique d'un texte non filmique, et n'aspire pas à autre chose. Et en ce sens, le bonimenteur-conférencier, par son texte à lui, creuse encore plus la distance qui peut séparer le spectateur du monde «diégétique» qui s'anime sous ses yeux. Il est alors dans une position similaire à celle du prêcheur dans sa chaire : une sorte d'instance posée comme en retrait d'un récit qui s'est produit «ailleurs» et «avant». Tel un intermédiaire entre le monde sacré et le monde profane, c'est entre la nef et le chœur, soit dans la chaire, que le prêtre se tient pour livrer son interprétation des textes sacrés. Tel un intermédiaire entre le monde diégétique et le monde «afilmique», pour reprendre l'expression de Souriau ${ }^{23}$, c'est entre la toile et la salle que le bonimenteur se tient pour livrer son interprétation de ces images plus filmées (noter le passé) que filmiques.

Ce sont d'ailleurs vraisemblablement ces similitudes qui ont servi, au Québec, à légitimer le cinéma, du moins au tout début. 
Ainsi, les premières années de leur exploitation du cinématographe en sol canadien (à compter, rappelons-le, de 1897), le vicomte Henry de Grandsaignes d'Hauterives et sa mère, Marie de Kerstrat (dite la Comtesse de Grandsaignes d'Hauterives) avaient-ils l'habitude de faire cautionner leur exploitation en insistant sur l'aspect «conférence» de leur représentation, au détriment de son aspect plus «spectacle». Ainsi de cette annonce, intitulée précisément «Conférences illustrées», parue en 1901 :

La Comtesse de Grandsaignes d'Hauterives, propriétaire de l'Historiographe qui voyage au Canada, avec son fils depuis 4 ans, offrant le concours de ses conférences illustrées à toutes les œuvres religieuses, prie les personnes qui auraient des doutes sur son identité et sa respectabilité de se renseigner près du consul de France («Conférences illustrées», La Presse, Montréal, 22 avril 1901).

Mais ces similitudes entre le prêcheur et le bonimenteur vont finir par jeter du discrédit sur le cinéma, qui sera longtemps au Québec honni du clergé. Du moins jusqu'à l'arrivée de ces prêtres-cinéastes qui ont été à la fois les "pionniers» d'un cinéma «made in Québec» et nos derniers bonimenteurs de vues animées. À l'instar de l'exploitant ambulant du début du siècle, ces prêtres sillonneront en effet, jusqu'à la Révolution tranquille, les campagnes de la «Belle Province» avec des films muets qu'ils auront eux-mêmes tournés et qu'ils accompagneront de leurs commentaires. Certains d'entre eux, Mgr Albert Tessier notamment, sont maintenant considérés comme de véritables cinéastes et, même, des poètes de l'image.

Pendant ce temps, hors de nos campagnes, le cinéma continuait son petit bonhomme de chemin, qui passait par l'élimination du bonimenteur. Mais il fallait, pour que ce bonimenteur disparût, que l'institution cinématographique finisse par se trouver une voie qui exclut toute prestation actorielle en direct, une voie qui laisse parler les seules images. Cette manière toute cinématographique d'injecter de la narration dans des images monstratives, ce moyen d'ajouter à la monstration des images une forme non verbale de narration, de remplacer la narration verbale du bonimenteur ou, plutôt, d'intégrer cette narration dans la bande-image même, ce fut le montage, pratique proprement discursive, mais iconique, qui a permis de développer un nouveau type de discours narratoriel simultané à la monstration, en substitution au discours narratoriel verbal du bonimenteur. Le montage, qui était en fait un moyen privilégié pour invisibiliser le narrateur, en l'intégrant à la bande-image, 
en l'aspirant dans le film, condition essentielle pour éliminer cette distance «primitive» à laquelle le commentaire du bonimenteur ne pouvait faire autrement que de donner lieu. Condition essentielle, finalement, pour que le spectateur puisse «enfin» suivre le même chemin que le bonimenteur et se fasse aspirer, à son tour, dans la fiction, dans cette fiction dont le contrôle était «enfin» passé totalement entre les mains du producteur, au détriment de l'exploitant dont le rôle ne serait plus jamais celui d'«interprète» du film.

\section{Université de Montréal}

\section{NOTES}

1 Voir André Gaudreault, Du littéraire au filmique. Système du récit (Paris/Québec : Méridiens Klincksieck/Presses de l'Université Laval, 1988).

2 Au Livre III de la République.

3 Le pionnier en la matière est assurément le chercheur américain Martin Sopocy qui a fort précocement écrit un article dans lequel il est frontalemént question du bonimenteur (identifié comme «narrateur») jusque dans le titre même de l'article. Voir "Un cinéma avec narrateur. Les premiers films narratifs de James A. Williamson", Les Cahiers de la Cinémathèque, Perpignan, no 29 (hiver 1979), pp. 108-126. L'article, écrit en 1977, a été distribué aux participants au fameux Congrès de la fédération internationale des archives du film sur le cinéma des premiers temps qui s'est tenu à Brighton (G.-B.) en mai 1978, et est ensuite paru, dans sa version originale anglaise, à l'automne 1978, dans la revue américaine Cinema Journal. On y lit notamment ce qui suit (pp. 110-111 de la version française) : «L'autobiographie de Cecil Hepworth ainsi que plusieurs autres sources indiquent clairement que celui qui présentait ces films en accompagnait la projection de nombreux commentaires. Il s'agissait généralement d'un projectionniste de métier, et la narration qu'il faisait durant la représentation était une pratique courante lors des projections de lanterne magique. Il n'existe aucune preuve démontrant que cette pratique ait été abandonnée par les premiers présentateurs d'images animées de l'Angleterre, qui avaient pour la plupart fait leurs débuts avec les projections de lanterne magique. Je suis d'avis que cette pratique s'est continuée plus longtemps que les historiens ne l'ont supposé — au moins jusqu'en 1903 — et que les films de cette période montrent une évidente dépendance vis-à-vis de cette narration verbale». Comme on le verra dans la suite du présent article, les faits historiques récemment redécouverts par les chercheurs montrent que les supputations de Sopocy étaient même en deçà de la réalité et que la pratique du boniment d'accompagnement a largement dépassé l'année 1903.

4 Les documents de la sorte à nous être restés sont d'autant plus rares qu'il s'agit là d'une pratique qui ne s'est, semble-t-il, jamais généralisée.

5 Comme le démontrent les recherches sur le sujet menées récemment par l'équipe que je dirige dans le cadre du projet «Les débuts du cinématographe au Québec», subventionné par le Conseil de recherche en sciences humaines du Canada (CRSH) et l'Université de Montréal. Cette recherche, menée en collaboration avec Germain Lacasse, a comme objectif de 
faire l'histoire des débuts de l'exploitation cinématographique au Québec, entre 1895 et 1915, par le truchement d'une étude du discours de la presse à son endroit.

6 Où, comme on sait, le benshi a joué, jusque vers la fin des années 30 , un rôle tout à fait particulier. Voir à ce sujet Noël Burch, Pour un observateur lointain. Forme et signification dans le cinéma japonais (Paris : Cahiers du cinéma/Gallimard, 1982).

7 Voir à ce sujet «L'écran ventriloque», dossier préparé sous la direction d'André Gaudreault, en collaboration avec Germain Lacasse, 24 images no 65 (Montréal, février-mars 1993) pp. 41-44.

8 Le mérite de la découverte de ce personnage fabuleux de l'histoire du cinéma au Québec revient entièrement à Germain Lacasse qui, alors qu'il était chercheur indépendant, a publié deux ouvrages essentiels sur le sujet. Voir Germain Lacasse (en collaboration avec Serge Duigou), L'Historiographe. Les débuts du spectacle cinématographique au Québec (Montréal : Cinémathèque québécoise, 1985). Voir également Serge Duigou et Germain Lacasse, Marie de Kerstrat. L'Aristocrate du cinématographe (Éditions Ressac, 1987). Marie de Kerstrat était la mère d'Henry de Grandsaignes.

9 C'est-à-dire les films composés d'un seul plan, qui sont restés majoritaires jusqu'aux environs de 1902.

10 C'est-à-dire les films composés de plusieurs plans.

11 C'est en raison d'une erreur typographique, semble-t-il, que le mot «jeu» se lisait «peu». Pour le bénéfice des lecteurs du présent article, nous nous sommes permis d'apporter la correction, entre crochets.

12 On peut cependant imaginer que les descriptions des catalogues étaient souvent conçues dans l'optique du boniment à faire. Il est en effet difficile de croire que les détails, fort nombreux, que de telles descriptions comprennent n'étaient là que pour permettre à l'éventuel acheteur (soit l'exploitant) de faire le choix des films devant composer son prochain programme. Par ailleurs, dans tous les cas où le boniment se basait sur un texte écrit (texte explicitement fourni à cette fin ou description de catalogue), on peut présumer que la part d'improvisation était grande.

13 À ce sujet, voir l'excellent article de Charles Musser, «Les Passions et les Mystères de la Passion aux États-Unis (1880-1900)» in R. Cosandey, A. Gaudreault et T. Gunning (direction), Une invention du diable? Cinéma des premiers temps et religion/ An Invention of the Devil? Religion and Early Cinema (Québec/Lausanne : Presses de l'Université Laval/Payot-Lausanne, 1992) pp. 145-186.

14 Faites à partir de photographies qui provenaient bien souvent d'Oberammergau en Bavière où, comme on sait, il était de tradition de jouer sur scène, à tous les dix ans, une Passion qui mettait à contribution l'ensemble des villageois.

15 Précisons que le mot «tableau» était en usage à la toute première époque du cinéma des premiers temps pour désigner le «plan», la «vue» ou le «film».

16 Ce terme a été suggéré par Alain Laframboise (p. 50).

17 Claude Gandelman, Le Regard dans le texte. Image et écriture du Quattrocento au XXe siècle (Paris : Méridiens Klincksieck, 1986) p. 30. Gandelman parle d'ailleurs du geste du «montreur» comme d'un «geste de la monstration", ce qui s'accorde bien avec les propositions que j'ai faites, pour le cinéma. 
18 Alain Laframboise (p. 52) nous apprend que cette hypothèse, avec laquelle il est en accord, lui vient de Michael Baxandall, Painting and Experience in Fifteenth-Century Italy (London : Oxford University Press, 1976).

19 Voici ce qu'écrit Alberti (cité par Alain Laframboise, p. 49) pour décrire dans son traité, sur un mode relativement normatif, la fonction de l'admoniteur : «Et il me plaît qu'il y ait dans l'istoria quelqu'un qui nous avertisse (ammonisca) et nous enseigne ce qui s'y fait, ou qui nous invite de la main à voir, ou qui nous menace avec un visage fâché et les yeux épouvantés afin que personne n'approche, ou qui nous invite à pleurer ou à rire avec eux.» C'est précisément en raison de l'utilisation par Alberti du mot ammonisca qu'Alain Laframboise a suggéré le terme «admoniteur».

20 Du moins, pour le bonimenteur, dans les cas où celui-ci se tenait au devant de la salle, ce qui, dans l'état actuel de nos recherches, semble le cas le plus fréquent.

21 Interviewé récemment par Germain Lacasse dans le cadre des recherches que nous menons, le célèbre homme de théâtre québécois Jean Grimaldi, qui se rappelle avoir vu des «bonimenteurs» à l'œuvre à Montréal encore à la toute fin des années 20, disait de ce «narrateur» (il utilisait d'ailleurs plus spontanément ce mot) qu'il «interprétait» les films.

22 Le mot «tableau» se prête bien à cette autre comparaison (voir, supra, la note 15).

23 Voir Étienne Souriau, L'Univers filmique (Paris : Flammarion, 1953).

\section{OUVRAGES CITÉS}

«Conférences illustrées». La Presse (Montréal, 22 avril 1901).

«Dans les Bois-Francs». L'Écho des Bois-Francs (Victoriaville, 3 mars 1900).

«Historical Cablo». Quebec Daily Mercury (Québec, 30 avril 1898).

«L'Historiographe à Joliette». L'Étoile du Nord (Joliette, 31 janvier 1901).

«L'Historiographe». Le Courrier de St-Hyacinthe (St-Hyacinthe, 29 octobre 1898).

Burch, Noël. «Porter ou l'ambivalence», in Raymond Bellour (direction), Le Cinéma américain, t. I. Paris : Flammarion, 1980.

Casetti, Francesco. D'un regard à l'autre. Le film et son spectateur. Lyon : Presses universitaires de Lyon, 1990.

Labrosse, Raoul. «Le Cinématographe». Les Annales térésiennes (SteThérèse, novembre 1900).

Laframboise, Alain. Istoria et théorie de l'art. Italie, XVe, XVIe siècles. Montréal : Presses de l'Université de Montréal, 1989.

Laroche, Maximilien. "Le Conte haïtien à la croisée des chemins». Actes du CELAT no 5, Université Laval, Québec (novembre 1989) p. 67.

Marsot, Gaëtane. L'Admoniteur d'Alberti et la figure mystérieuse de Pino : leur fonction dans l' «istoria». Mémoire de maîtrise, Département d'histoire de l'art, Université de Montréal, 1985.

Velay-Vallantin, Catherine. L'Histoire des contes. Paris : Fayard, 1992. 